\title{
Extensional rheometry of model liquids: Simulations of filament stretching
}

\author{
Hassager, Ole; Wang, Yanwei ; Huang, Qian
}

Published in:

Physics of Fluids

Link to article, DOI:

$10.1063 / 5.0076347$

Publication date:

2021

Document Version

Peer reviewed version

Link back to DTU Orbit

Citation $(A P A)$ :

Hassager, O., Wang, Y., \& Huang, Q. (2021). Extensional rheometry of model liquids: Simulations of filament stretching. Physics of Fluids, 33, [123108]. https://doi.org/10.1063/5.0076347

\section{General rights}

Copyright and moral rights for the publications made accessible in the public portal are retained by the authors and/or other copyright owners and it is a condition of accessing publications that users recognise and abide by the legal requirements associated with these rights.

- Users may download and print one copy of any publication from the public portal for the purpose of private study or research.

- You may not further distribute the material or use it for any profit-making activity or commercial gain

- You may freely distribute the URL identifying the publication in the public portal

If you believe that this document breaches copyright please contact us providing details, and we will remove access to the work immediately and investigate your claim 


\section{Extensional rheometry of model liquids: Simulations of filament stretching}

Ole Hassager, ${ }^{1, \text { a) }}$ Yanwei Wang (王衍伟), ${ }^{2,3}$ and Qian Huang (黄茜) ${ }^{4}$

1) Danish Polymer Center, Department of Chemical and Biochemical Engineering, Technical University of Denmark, 2800 Kgs. Lyngby, Denmark

${ }^{2)}$ Department of Chemical and Materials Engineering, School of Engineering and Digital Sciences, Nazarbayev University, Nur-Sultan 010000, Kazakhstan

3) Laboratory of Computational Materials Science for Energy Applications, Center for Energy and Advanced Materials Science, National Laboratory Astana, Nur-Sultan 010000, Kazakhstan

4) Polymer Research Institute, Sichuan University, Chengdu 610065, China

(Dated: 29 November 2021)

Polymeric liquid bridges are known to fail during extension. This phenomenon of failure seemingly calls into question the operating principle of filament stretching rheometers. In these devices, a polymeric sample is initially placed between two plates. The sample is then stretched into a liquid bridge by moving the plates apart in a specified way. We here show that a steady extensional viscosity can be measured if the plates are separated in such a way that the stretch rate in the filament symmetry plane is kept constant, even for liquids highly prone to instability. Moreover, reliable measurements of the stress during a relaxation phase can be obtained as well. The conclusions are based on simulations for a number of constitutive equations, including the Newtonian liquid, the Oldroyd-B model, the differential non-stretch Rolie-Poly model, and the integral Doi-Edwards model with and without associated stretch relaxation dynamics.

a) Author to whom correspondence should be sent: Ole Hassager, email: oh@kt.dtu.dk 


\section{INTRODUCTION}

\section{A. Continuum vs. Molecular descriptions}

Recognizing the importance of understanding the dynamics of polymeric liquid, R.B. Bird took the lead in establishing a combination of textbooks ${ }^{1,2}$ that encompass both the fluid mechanics and the molecular theory of polymeric liquids. Starting with the model for Newtonian fluids, volume I describes several primarily nonlinear constitutive equations and the macroscopically observable flow patterns that result from given boundary conditions such as pressure drop or imposed macroscopic displacements. Molecules do not appear explicitly, but the techniques are to be used, for example, in the design of polymer processing operation based on a rheological characterization of the specific material. Volume II, on the other hand, is devoted ultimately to the derivation of constitutive equations from a knowledge of the underlying molecular structure. The assumption here is that even in an inhomogeneous flow field, all that a nanometer-size molecule can ever cover is a homogeneous flow field. The resulting viscosity functions, or better yet complete constitutive equations, can then be compared to experimental measurements in well-defined homogeneous flows, be they shear or elongation. The constitutive equations alone do not allow conclusions on macroscopic flow effects such as instability or recirculations. Yet while the two volumes describe two disjoint disciplines, an underlying appreciation of both disciplines is sometimes needed to resolve problems that cannot be resolved from either one alone. One such problem is related to the analysis of instrumentation for extensional rheology by means of the stretching of liquid polymer filaments. A key motivation for the stretching of polymer liquid filaments is to gain insight into the molecular dynamics in extensional flow. One topic of current interest is that of monomeric friction reduction in strong flows ${ }^{3,4}$. Clearly, in order to make any molecular inferences from the macroscopic stretching of liquid filaments, it is vital to perform the measurements at well-defined and controlled stretch rates. It is to this latter question that the present work is devoted.

\section{B. Fluid mechanics of filament stretching}

The stability of a liquid filament has been the object of substantial investigations. It is known, for example, that cohesive forces in the form of surface tension promote the breakup of jets ${ }^{5}$. Conversely, it was demonstrated by Renardy ${ }^{6}$ that a Newtonian liquid filament in the absence of surface tension could be extended to any imposed length without rupture. Yet even 
in the absence of surface tension, elastic forces can drive an instability in a liquid filament that eventually leads to rupture. This has been described and analyzed by Malkin and Petrie ${ }^{7}$ who formulated a master curve for the nominal Hencky strain that may be obtained for a filament of monodisperse linear polymers. This curve has also been observed for a range of monodisperse linear polymers by Zhu and Wang ${ }^{8}$.

The phenomenon of rupture due to filament necking has been modeled by finite element simulations for a specific integral constitutive equation ${ }^{9}$. While rupture by necking can be simulated for one isolated model, that does not cast light on the underlying stability modes. In a recent series of landmark publications ${ }^{10-12}$, Hoyle and Fielding have derived criteria for extensional necking instability in the stretching of liquid filaments. Specifically for the start-up of constant strain rate extensional flow, Hoyle and Fielding derived two criteria and associated necking modes: The so-called "Stress curvature mode" and the "Elastic Considère mode". Hoyle and Fielding illustrated both of these modes for a wide range of constitutive equations ranging from the highly strain-hardening Upper Convected Maxwell model to the extremely strainsoftening nonstretching Rolie-Poly model. The two modes act rather differently with respect to the wavelength of the resulting deformation, with the elastic Considère mode having the shorter wavelength.

For most complex fluids undergoing start-up of constant strain-rate, the Hoyle and Fielding criteria predict ${ }^{10-12}$ that a necking instability will occur at a relatively low Hencky strain. In apparent contrast to this, the technique of Filament Stretching Rheometry (FSR) ${ }^{13,14}$ is routinely used to characterize the nonlinear extensional properties of complex liquids including their steady extensional viscosity ${ }^{15-18}$. Fortunately, the conflict is merely apparent. The now established Filament Stretching Rheometers do not impose a constant overall rate of separation of the plates. Instead, the controlled Filament Stretching Rheometers move the plates such that the stretching rate in the symmetry plane between the two plates has a desired time history. Obtaining a desired kinematic history in the symmetry plane by moving the endplates is not necessarily a simple procedure. Thus for each new liquid, Orr and Sridhar ${ }^{19}$ experimentally determined a relation between the specified nominal Hencky strain (based on the plate separation) and the resulting true Hencky strain (based on the mid-filament diameter). They could then invert this relation to establish the plate separation history needed to obtain a desired true Hencky strain history for a range of polymer solutions. Bach, Rasmussen, and Hassager ${ }^{16}$ utilized the Orr-Sridhar technique for polymer melts but found that they needed up to three iterations before a satisfactory true Hencky strain history was obtained for a specific 
polyethylene melt. The next improvement in experimental FSR was the development of online control systems ${ }^{20,21}$ that do enable a desired true Hencky strain history to be established in the filament symmetry plane in real-time. It seems natural, therefore, to analyze what consequences the Hoyle-Fielding stability criteria may have for controlled FSR. Both differential and integral models will be used. Since Hoyle and Fielding applied ${ }^{10-12}$ their analysis to a large number of differential models, it seems natural to begin with this group of models. The differential models will be analyzed in the Hoyle-Fielding Eulerian frame. Then the analysis turns to integral models for which a Lagrangian frame seems more easily applicable.

Before proceeding with the FSR analysis, a comment is in place on the seemingly related Capillary Breakup Extensional Rheometer (CaBER) technique ${ }^{22-25}$. In the CaBER the user cannot set the stretch rate prior to the experiment. The CaBER instrument will choose its own strain rate history from a balance between liquid surface tension and rheology. The user may then use the time-varying instantaneous stretch rate to extract an apparent extensional viscosity $^{22}$. By contrast, the user of a controlled FSR imposes a given strain rate history on the liquid in the mid-filament plane. For example, the user may wish to determine the steady extensional viscosity at a given rate by imposing a constant rate. The key question for the FSR, therefore, is if the stress becomes steady before the liquid bridge fails due to one of the Hoyle-Fielding instability modes. A second important question relates to the measurement of stress relaxation in a filament after stretching. Without online control, viscoelastic filaments frequently fail due to a delayed necking phenomenon. The question, therefore, is if incorporation of on-line control can avoid the delayed necking phenomenon.

\section{DEFINITION OF STRAIN MEASURES}

To be more explicit with the notation, consider a material between two parallel plates (see Figure 1 for photo and sketch) separated initially by the distance $L_{0}$ and at a later time $t$ by the distance $L(t)$. The nominal Hencky strain is then by definition

$$
\epsilon_{N}(t)=\ln \left(\frac{L}{L_{0}}\right)
$$

Assume moreover that the liquid bridge between the plates is symmetrical around the midfilament plane. Let the initial radius in the mid-filament plane be $R_{0}$ and the radius at time $t$ be $R(t)$. The true Hencky strain of the material in the mid-filament plane is then ${ }^{26}$

$$
\epsilon(t)=2 \ln \left(\frac{R_{0}}{R}\right)
$$


What one imposes in an experimental design is the plate motion, i.e., a given function $\epsilon_{N}(t)$.

The master curve by Malkin and Petrie, the analysis by Hoyle and Fielding ${ }^{10-12}$ and in fact, most commercially available extensional rheometers apply a constant value of the nominal Hencky strain rate, $d \epsilon_{N} / d t$.

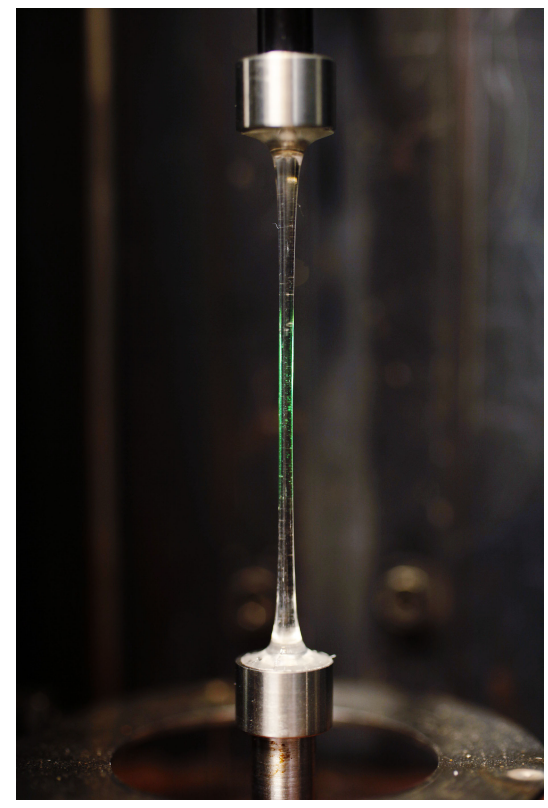

(a)
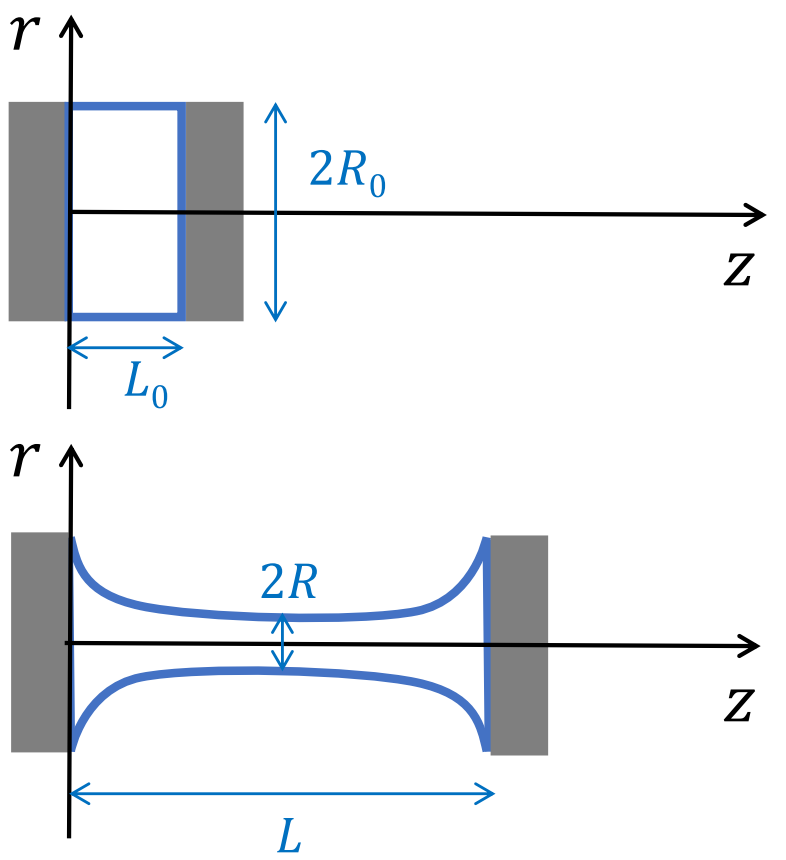

(b)

FIG. 1: (a) Photo of stretched polystyrene melt with the stretching axis vertical (Copyright 2019 The American Physical Society) ${ }^{27}$. (b) Cylindrical coordinate system $(r, z)$ used in this work with the $z$-axis horizontal.

By contrast, the controlled filament stretching rheometers impose a plate motion $\epsilon_{N}(t)$ such that a desired true Hencky strain, $\epsilon(t)$, is obtained in real-time. This could be, for example, a constant value of the true Hencky strain rate $\dot{\epsilon} \equiv d \epsilon / d t$, but it could also be $\dot{\epsilon}=0$ such as is needed to measure stress decay in a quiescent liquid due to molecular relaxation. All results in the present computational rheology study ${ }^{28}$ have been obtained by the imposition of such a control scheme $\mathrm{s}^{20,21}$. The detailed operation of the control loop is not the attention of the present work, but the degree to which the control is successful is evaluated by comparing the actual $\epsilon(t)$ with the prescribed function of time. 


\section{DIFFERENTIAL MODELS AND EULERIAN SIMULATIONS}

Following Hoyle and Fielding ${ }^{10}$, the liquid bridges are simulated with an application of the thin filament approximation in an Eulerian frame. The independent variables are the axial coordinate $z$ and time $t$. A stretching transformation is implemented such that the axial coordinate $z$ is mapped into a corresponding variable $u \in[0 ; 1]$. The dependent variables are the filament radius, filament velocity, and a configurational quantity $Z$ as further detailed in

\section{Appendix A.}

\section{A. Newtonian liquids}

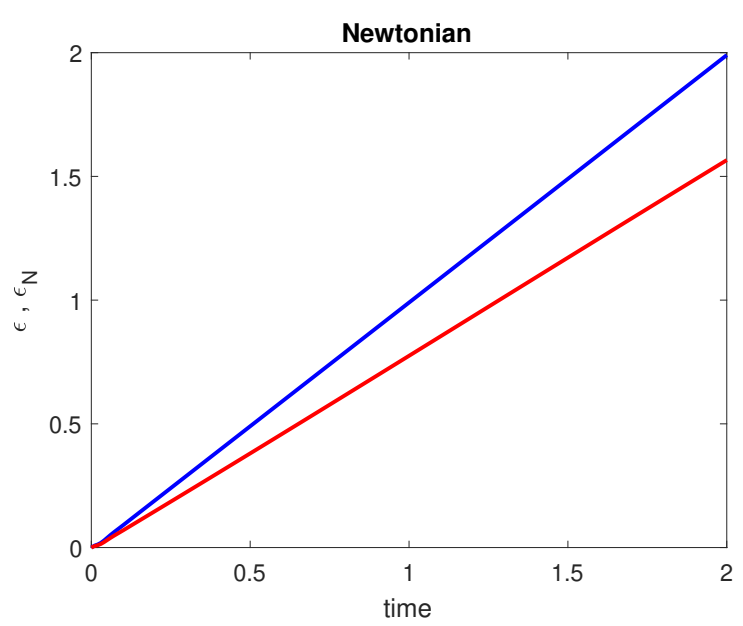

(a)

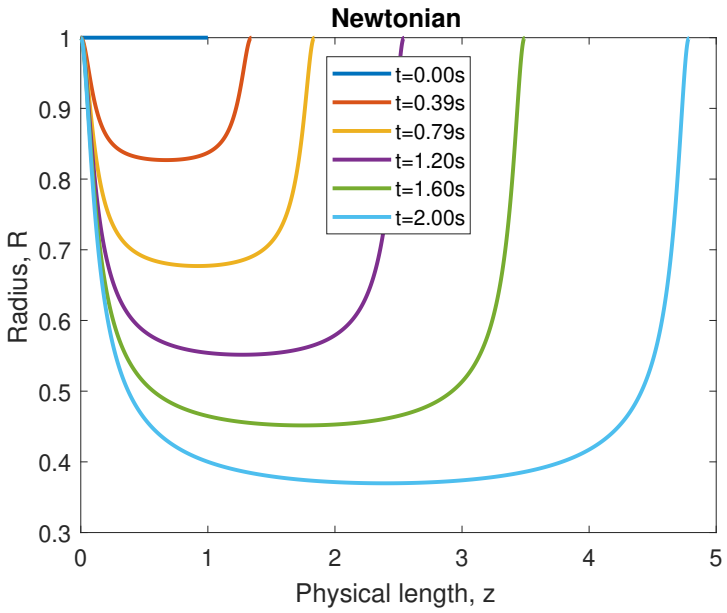

(b)

FIG. 2: Slender filament simulations of controlled constant rate stretching of a Newtonian liquid. (a) True (top, blue) and nominal (lower, red) Hencky strains as function of time. (b) Radial profiles for $t=0.00,0.39,0.79,1.20,1.60$, and 2.00, in units of inverse stretch rate, which for convenience was set to $1 \mathrm{~s}$.

Hoyle and Fielding define a "stress curvature mode" of instability ${ }^{10}$. This mode is predicted to set in whenever the second derivative of the axial stress with respect to time is negative. Strictly speaking, this would not occur for a perfectly controlled filament stretching of a Newtonian liquid, since in that case, the axial stress is constant in time. However, given that the stress starts out at zero and then (almost) instantaneously increases to the steady value, one can argue that in the process the second derivative must have been negative (however briefly). This would then also be in line with predictions for the viscoelastic models that are invariably "stress 
curvature" unstable at zero Weissenberg numbers.

To introduce notation, consider a Newtonian liquid of viscosity $\eta_{s}$. The extra stress $\boldsymbol{\sigma}$ is then given by

$$
\boldsymbol{\sigma}(t)=\eta_{s} \dot{\gamma}(t)
$$

where $\dot{\gamma}=\boldsymbol{\nabla} \boldsymbol{v}+(\boldsymbol{\nabla} \boldsymbol{v})^{\dagger}$ denotes the rate-of-strain tensor. The stress is defined as positive in tension (opposite to the notation $i^{1,2}$ ) while the definition of the rate-of-deformation tensor $\dot{\gamma}$ is identical to that of the $\mathrm{DPL}^{1,2}$. The results from simulations of a controlled filament stretching experiment are shown in Figure 2. The simulations show a linearly increasing true Hencky strain up to a Hencky strain of 2 as marked in blue in Fig. 2a. The corresponding nominal Hencky strain dictated by the control loop is shown in red. The radial profiles shown in Fig. 2 b develop a rather wide neck. The constant value of $\dot{\epsilon}$ is demonstrated by the constant slope of $\epsilon$ in Fig. 2a. Conversely it is seen that $\epsilon_{N}$ lags behind $\epsilon$ by almost half a Hencky strain unit when $\epsilon=2$. Hence online control is mandatory to obtain accurate measurements for Newtonian liquids.

The Newtonian liquid is interesting since we also have the result by Renardy ${ }^{6}$ that (in the absence of surface tension) the filament will not fail in finite time. This might indicate that the "stress curvature" mode is not a very dangerous mode for rheometry. That is also implied by Hoyle and Fielding ${ }^{10}$ who on the stress curvature mode stated that "Once active, it causes necking with a relatively gentle rate of development."

\section{B. Oldroyd-B and the non-stretching Rolie-Poly toy model}

The effects of liquid elasticity are illustrated with the infinitely stretchable Oldroyd-B model $^{1,2}$ on the one hand and the non-stretchable Rolie-Poly model ${ }^{29,30}$ as the other extreme. Both models are roughly ${ }^{31}$ incorporated in the following toy model:

$$
\begin{gathered}
\frac{d \mathbf{W}}{d t}=\boldsymbol{\kappa} \cdot \mathbf{W}+\mathbf{W} \cdot \boldsymbol{\kappa}^{\dagger}-\tau_{d}^{-1}(\mathbf{W}-\boldsymbol{\delta})-\beta \boldsymbol{\kappa}: \mathbf{W} \cdot(\mathbf{W}-\boldsymbol{\delta}) \\
\boldsymbol{\sigma}=G_{0} \mathbf{W}+\eta_{s} \dot{\gamma}
\end{gathered}
$$

where $\kappa_{i j}=\partial v_{i} / \partial x_{j}, \mathbf{W}$ is a polymer conformation tensor, $G_{0}$ is a modulus, and $\tau_{d}$ is a polymer relaxation time that is used to scale time in Figs. 3 to 5 . The parameter $\eta_{s}$ is a Newtonian contribution to the stress often called a solvent contribution. The zero-shear-rate viscosity $\eta_{0}=G_{0} \tau_{d}+\eta_{s}$. Here and in the following the relative amount of solvent viscosity to total viscosity is $\eta_{s} / \eta_{0}$. The parameter $\beta$ is similar to the $\beta$ used by Hoyle and Fielding ${ }^{10}$ 
in their Rolie-Poly toy model. For $\beta=0$, the model reduces to the Oldroyd-B model ${ }^{1,2}$. The corresponding steady stress is shown in Fig. 4a with the Oldroyd-B showing the known stress singularity at a non-dimensional stretch rate 0.5 .

\section{Oldroyd-B}

Consider in Figure 3 the simulation results for stretching of the Oldroyd-B model at a Weissenberg number $\mathrm{Wi}=\dot{\epsilon} \tau_{d}=1$ up to a total strain $\epsilon=2$. At this rate, the filament does not suffer any of the two instability modes by Hoyle and Fielding. This is reflected in two observations. Firstly, the filament radius (as shown in Fig. 3b) is practically uniform along its entire length apart from adjustments near the plates. Secondly, the nominal and true Hencky strains follow closely one another as shown in Fig. 3a. This would, in some sense, be good news from the point of rheometry: The control loop is not needed. The downside, however, is that the stress increases without bound as $t \rightarrow \infty$. In other words, no steady extensional viscosity will be obtained.

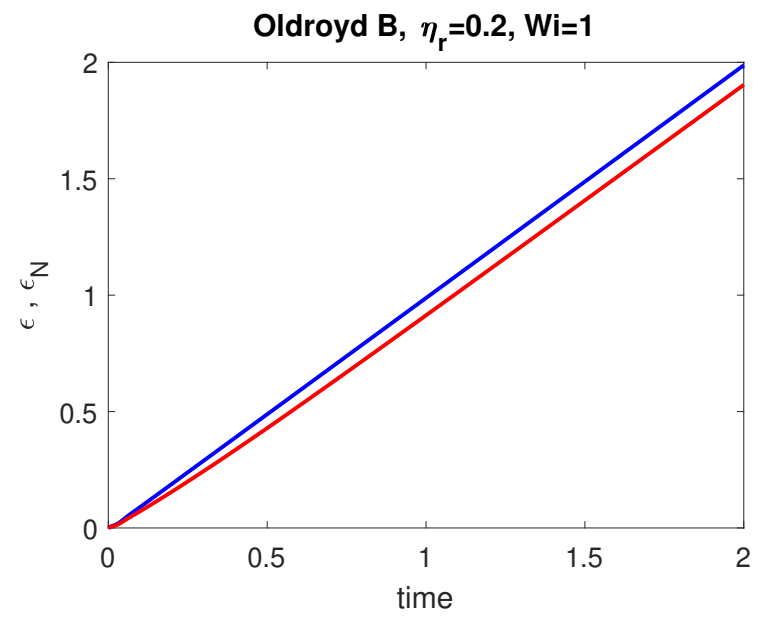

(a)

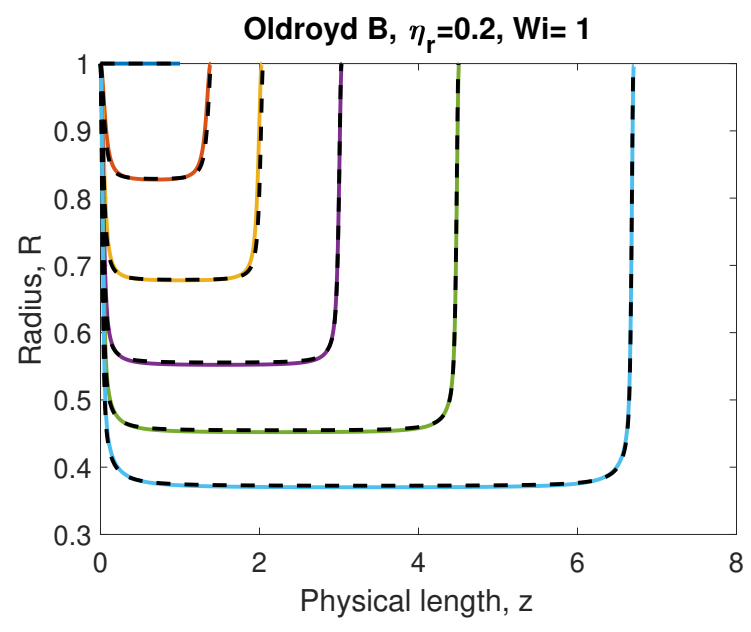

(b)

FIG. 3: Slender filament simulations of controlled constant rate stretching of the Oldroyd-B model. (a): True (top) and nominal (lower) Hencky strains as function of time. (b): Radial profiles for time (from top to bottom at $z=1$ ) 0.00, 0.40, 0.80, 1.20, 1.60 and 2.00, in units of $\tau_{d}$; Full (colored) lines are results from the Eulerian method; dashed (black) lines are from the Lagrangian method. 


\section{Rolie-Poly}

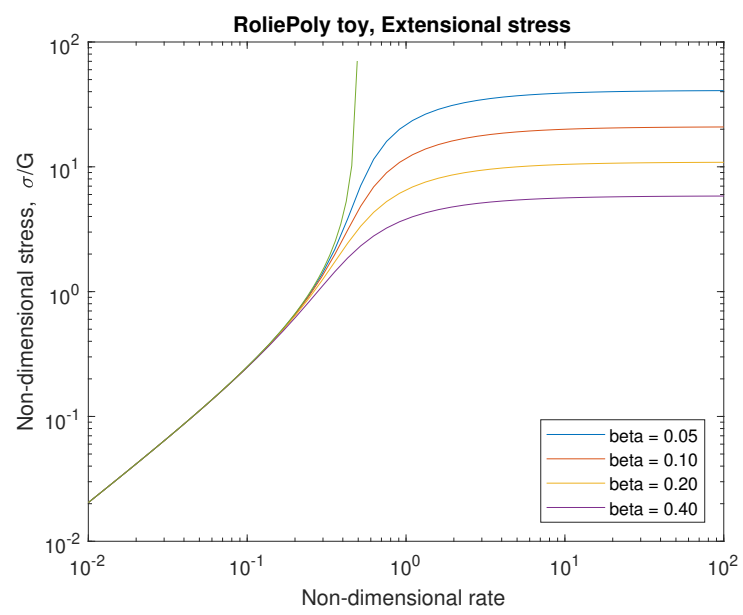

(a)

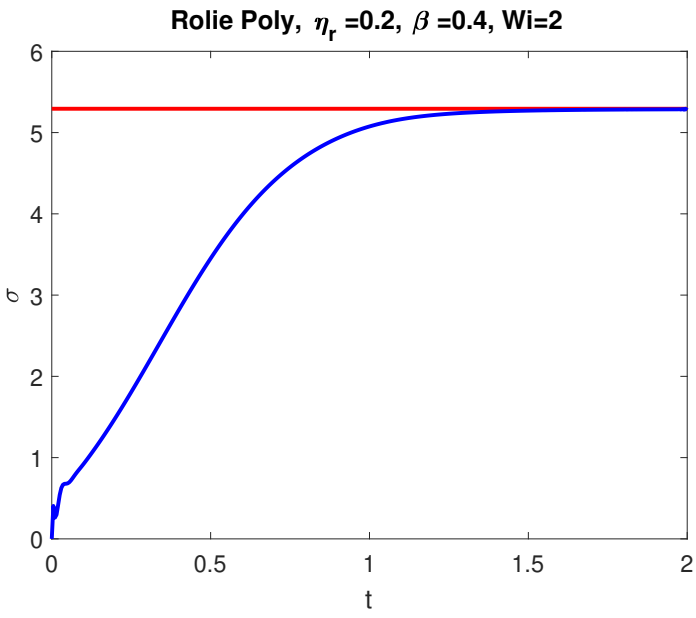

(b)

FIG. 4: (a): Steady extensional stress for the non-stretching Rolie-Poly model without solvent contribution. The green curve corresponds to the Oldroyd-B model $(\beta=0)$. (b): the blue curve indicates the stress in the middle of the filament in start-up of constant rate of the model for $\beta=0.4$ and $20 \%$ solvent viscosity, while the red curve indicates the analytical steady stress.

At the other extreme, we find the non-stretching Rolie-Poly model. For $\beta>0$ and no solvent viscosity the model displays an upper limit to the elastic stress in extension as shown in Fig. $4 \mathbf{a}$. The limit at infinite rate is given by the difference between axial and radial components of the W tensor as follows

$$
W_{z z}-W_{r r} \rightarrow(1+\sqrt{1+2 \beta}) / \beta \text { for } \mathrm{Wi}=\dot{\epsilon} \tau_{d} \rightarrow \infty
$$

This corresponds to a stress saturation as for the Doi-Edwards model. In fact, the Doi-Edwards model with no stretching shows ${ }^{32}$ a stress saturation of $5 G_{0}$. Following Hoyle and Fielding ${ }^{10}$, we use exclusively $\beta=0.4$, corresponding to an elastic saturation of about $5.8 G_{0}$ (Fig. $\left.4 \mathbf{a}\right)$.

Figures $\mathbf{4 b}$ and $\mathbf{5}$ display the results of a start-up experiment with the non-stretching Rolie-Poly toy model at $\mathrm{Wi}=2$. At this rate, the Hoyle-Fielding conditions predict that the filament is unstable to both a "stress-curvature" instability and an "elastic Considère" instability $^{33}$. Fig. $\mathbf{4 b}$ records the stress in the symmetry plane as function of time (in units of $\tau_{d}$ ). The horizontal red line is the steady stress. It appears that the steady stress is obtained more or less at about $t=\tau_{d}$. The true and nominal Hencky strains as a function of time are shown in 


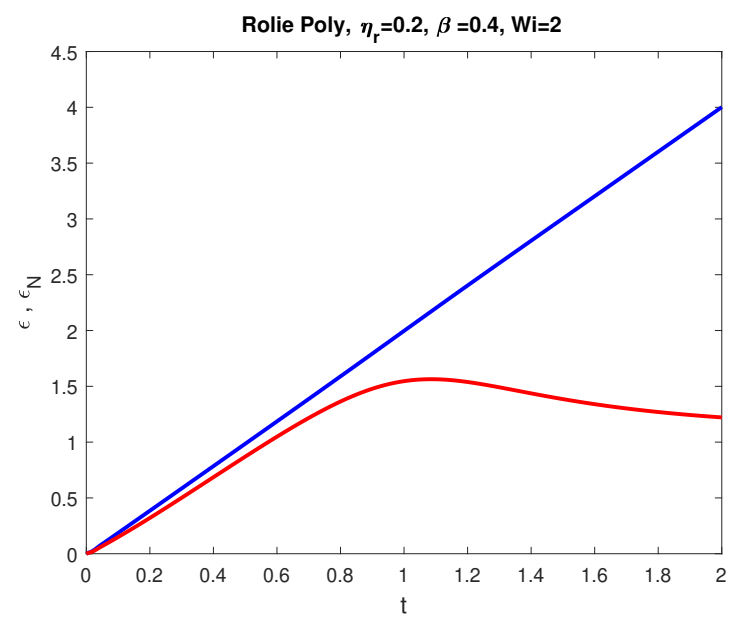

(a)

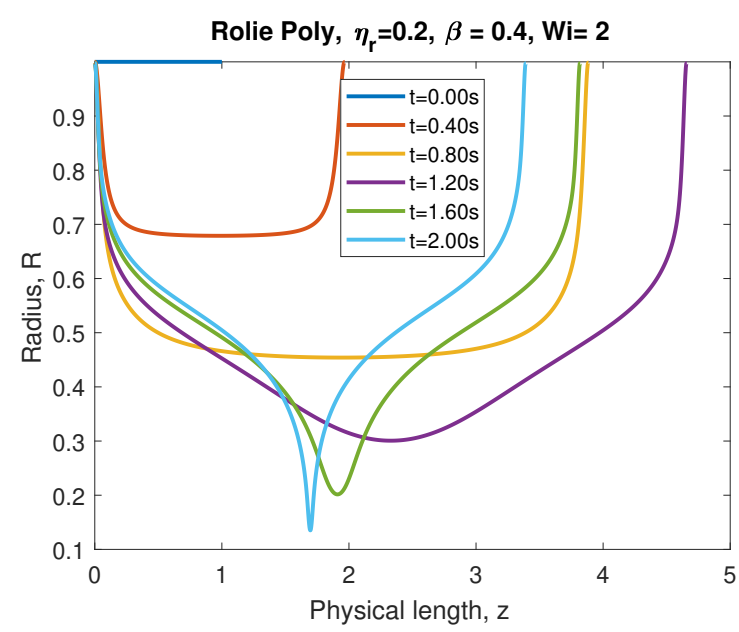

(b)

FIG. 5: Continuation of the slender filament simulation of controlled stretching of a non-stretching Rolie-Poly model in Fig. 4b. (a): True (top) and nominal (lower) Hencky strains as function of time. (b): Radial profiles for time 0.00, 0.40, 0.80, 1.20, 1.60, and 2.00, in units of $\tau_{d}$, which, for convenience, was set to $1 \mathrm{~s}$.

Fig. 5a. It appears that while the true Hencky strain in the symmetry plane increases linearly with time, that is not true at all for the nominal Hencky strain. On the contrary, from about $t=\tau_{d}$, the nominal Hencky strain decreases, which corresponds to the plates moving back towards each other. This backward motion may also be seen in Fig. 5b where the development of a very narrow neck in the filament is observed in the final stages of the stretching operation. This narrow neck is the result of the elastic Considère instability. The profile is similar to that observed by Bazzi and Carvalho ${ }^{34}$ in the break-up of liquid sheets. Such a narrow neck will eventually make it impossible to follow the mid-filament diameter in practice. In fact, even the simulations were terminated at this stage. However, it is important to note that the steady stress was obtained before the catastrophic development in filament shape. Thus with the feedback control, it is possible to measure the steady viscosity of a stress-saturation liquid such as the toy-Rolie-Poly model.

\section{INTEGRAL MODELS AND LAGRANGIAN SIMULATIONS}

Integral models are formulated most conveniently not in terms of the relaxation modulus $G(t)$ but rather in term of the memory function $M(t)=-(d / d t) G(t)$. Simulations will be shown for two different memory functions: 
- The simple single-mode Maxwell model

$$
M(t)=\frac{G_{0}}{\tau_{d}} \exp \left(-t / \tau_{d}\right)
$$

with modulus $G_{0}$ and the single relaxation time $\tau_{d}$.

- The continuous Baumgaertel-Schausberger-Winter (BSW) ${ }^{35,36}$ relaxation spectrum, defined by:

$$
\begin{gathered}
M(t)=\int_{0}^{\infty} H(\tau) \frac{\exp (-t / \tau)}{\tau^{2}} d \tau \\
H(\tau)=H_{e}(\tau)+H_{g}(\tau)
\end{gathered}
$$

where

$$
\begin{aligned}
& H_{e}(\tau)=n_{e} G_{0}\left(\frac{\tau}{\tau_{m}}\right)^{n_{e}} h\left(1-\tau / \tau_{m}\right) \\
& H_{g}(\tau)=n_{e} G_{0}\left(\frac{\tau}{\tau_{c}}\right)^{-n_{g}} h\left(1-\tau / \tau_{c}\right)
\end{aligned}
$$

Detailed meaning of the parameters is given by Huang et al. ${ }^{37}$. Specifically, the low frequency asymptote of the loss modulus is

$$
G^{\prime \prime}(\omega)=G_{0} \frac{n_{e}}{1+n_{e}} \tau_{m} \omega+\cdots
$$

In other words the upper limit of time constant $\tau_{m}$ is smaller than the equivalent single-mode Maxwell relaxation time $\tau$ by the factor $n_{e} /\left(1+n_{e}\right)$. Therefore the Weissenberg number for the BSW model is defined as $\mathrm{Wi}=\dot{\epsilon} \tau_{m} n_{e} /\left(1+n_{e}\right)$ in order that the Weissenberg numbers for the BSW model simulations can be directly compared with those of the previous calculations. Also, $\tau_{d}=\tau_{m} n_{e} /\left(1+n_{e}\right)$ (which, for convenience, was set to $1 \mathrm{~s}$ ) is used to scale time in the results shown in Figures 6, 7, and 8. Also, notice that in this work, we have used $G_{0}$ for the plateau modulus ${ }^{38}, \mathrm{G}_{\mathrm{N}}^{0}$, for convenience of writing.

\section{A. Oldroyd-B model revisited}

The integral version of the Oldroyd-B model reads:

$$
\boldsymbol{\sigma}(t)=\int_{t^{\prime}=-\infty}^{t} M\left(t-t^{\prime}\right) \mathbf{B}\left(t, t^{\prime}\right) d t^{\prime}+\eta_{s} \dot{\gamma}
$$

The memory function is given by Eq. $7, \eta_{s}$ is a solvent viscosity and $\mathbf{B}\left(t, t^{\prime}\right)$ is the Finger strain tensor $^{1,2}$ of the deformation from $t^{\prime}$ to $t$. The relative amount of solvent viscosity to total viscosity is

$$
\eta_{r}=\eta_{s} / \eta_{0}=\eta_{s} /\left(G_{0} \tau_{d}+\eta_{s}\right)
$$


The Weissenberg number is defined as $\mathrm{Wi}=\dot{\epsilon} \tau_{d}$. The independent variables are the material coordinates and time $t$. The material coordinates are conveniently taken as the initial axial positions along the filament. The dependent variables are the particle positions and function of time and the total tension in the filament also as a function of time. The scheme for the Oldroyd-B model is further detailed in Appendix B.

Figure $3 \mathbf{b}$ shows a comparison of the Lagrangian-based simulation scheme with the Eulerian scheme. The dashed black lines, obtained with the Lagrangian scheme, were superposed on the colored lines obtained with the Hoyle-Fielding Eulerian scheme. While this is comforting in that it gives faith in both schemes, it should be kept in mind that the Oldroyd-B model at $\mathrm{Wi}>0.5$ is not very interesting from a rheometric point of view since no steady flow is possible.

\section{B. Doi-Edwards toy model}

The fundamental nonlinear assumption in the Doi-Edwards model for linear polymers is that the primitive path of the chains orient in flow but do not stretch relative to the equilibrium length. A simple way to incorporate this idea in a continuum model is to replace the Finger strain tensor in Eq. 13 with the Finger strain tensor divided by its trace. This gives a model with stress saturation at large extensional rates similar to the original Doi-Edwards model ${ }^{32}$. To include the possibility that linear polymer molecules may stretch above their equilibrium primitive path, Pearson et al. ${ }^{39}$ introduced a molecular stretch variable, $\lambda(t)$, determined from an equation of the form:

$$
\frac{d}{d t} \lambda=\lambda(\boldsymbol{\kappa}: \mathbf{S})-\frac{1}{\tau_{s}} f(\lambda)(\lambda-1)
$$

where $\kappa_{i j}=\partial v_{i} / \partial x_{j}$, and the structure tensor is defined from the pure Doi-Edwards stress

$$
\boldsymbol{\sigma}(t)=3 G_{0} \lambda^{2} \mathbf{S}(t)+\eta_{s} \dot{\gamma}
$$

where

$$
\mathbf{S}(t)=\int_{t^{\prime}=-\infty}^{t} \frac{1}{G_{0}} M\left(t-t^{\prime}\right) \mathbf{Q}\left(t, t^{\prime}\right) d t^{\prime}
$$

$M\left(t-t^{\prime}\right)$ is the memory function, and

$$
\mathbf{Q}\left(t, t^{\prime}\right)=\frac{\mathbf{B}\left(t, t^{\prime}\right)}{\operatorname{tr}\left[\mathbf{B}\left(t, t^{\prime}\right)\right]}
$$

It will be referred to as the DE-toy model in the following. The governing equation for $\lambda$ contains the molecular stretch relaxation time $\tau_{s}$. The associated Weissenberg number is denoted $\mathrm{Wi}_{R}=$ 
$\dot{\epsilon} \tau_{s}$. Several choices can be made for the nonlinear function $f(\lambda)$ in Eq. 15. We use here the form suggested by Wagner and co-workers ${ }^{40}$,

$$
f(\lambda)=1-\frac{2}{3} p+\frac{2}{9} \lambda^{2}\left(\lambda^{2}+\lambda+1\right) p
$$

with the parameter $p=0.3$.

The definition of $\mathbf{S}(t)$ in Eq.17 is compatible with the single-mode Maxwell formulation of Blackwell et al. ${ }^{41}$. However, it is also used together with the BSW continuous relaxation spectrum.

\section{Stress saturation with no stretching}

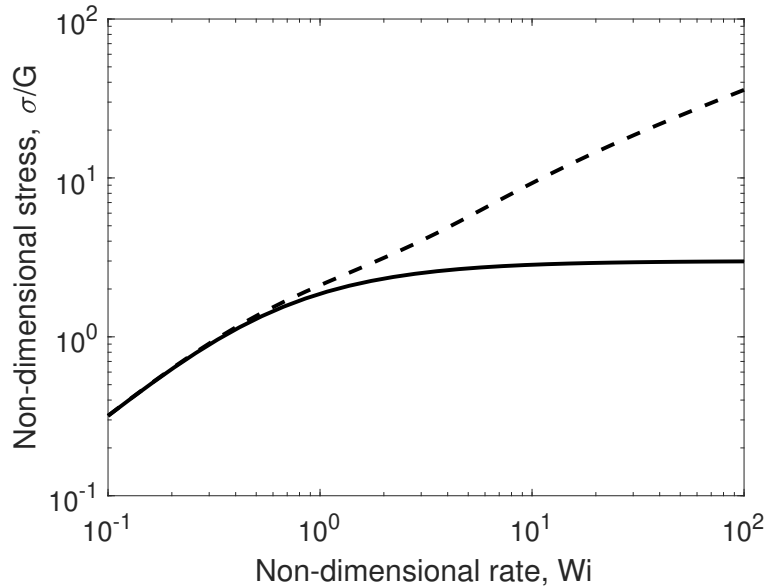

(a)

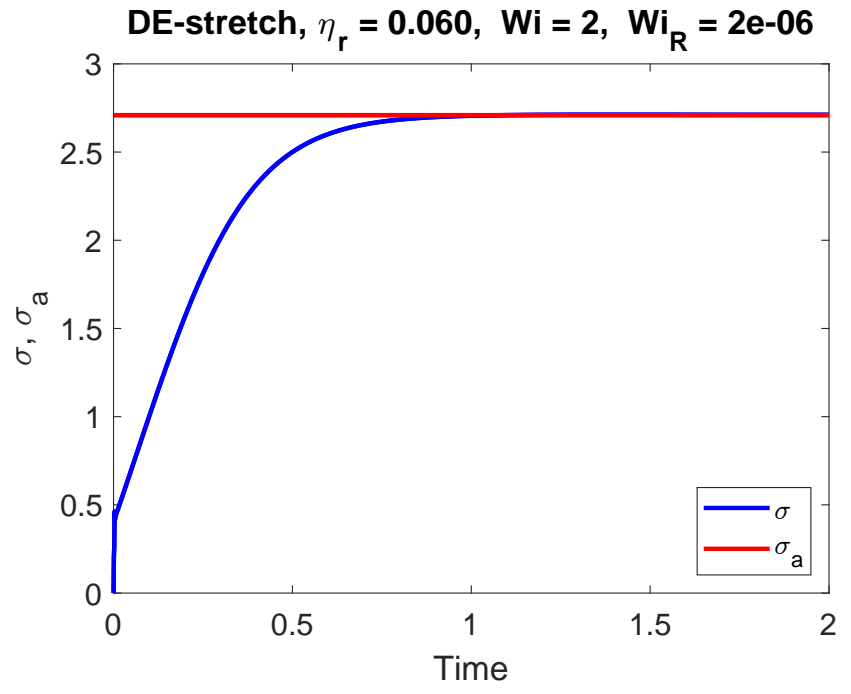

(b)

FIG. 6: (a): (full line) steady extensional stress for a non-stretching DE-toy model without solvent contribution; (dashed line) steady extensional viscosity for a stretching DE-toy model with $\mathrm{Wi}_{R}=0.1 \mathrm{Wi}$. (b): (blue curve) the extensional stress growth of single-mode (Eq. 7)

DE-toy model at $\mathrm{Wi}_{R} \ll 1$ with relative solvent contribution of $6 \%$; (red line) the corresponding analytical steady stress.

The Oldroyd-B model is the only differential model for which a direct comparison can be made between simulations in the Eulerian description and the Lagrangian description as formulated here. Yet, the non-stretching Rolie-Poly model and the non-stretching Doi-Edwards model share the feature of stress saturation. It was demonstrated that the Rolie-Poly model develops a highly localized neck as a result of the elastic Considère instability. It seems natural, 


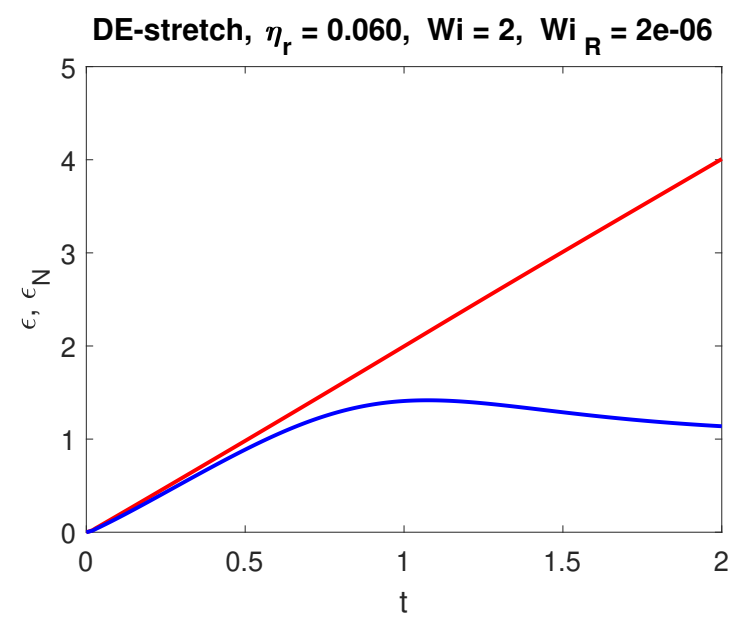

(a)

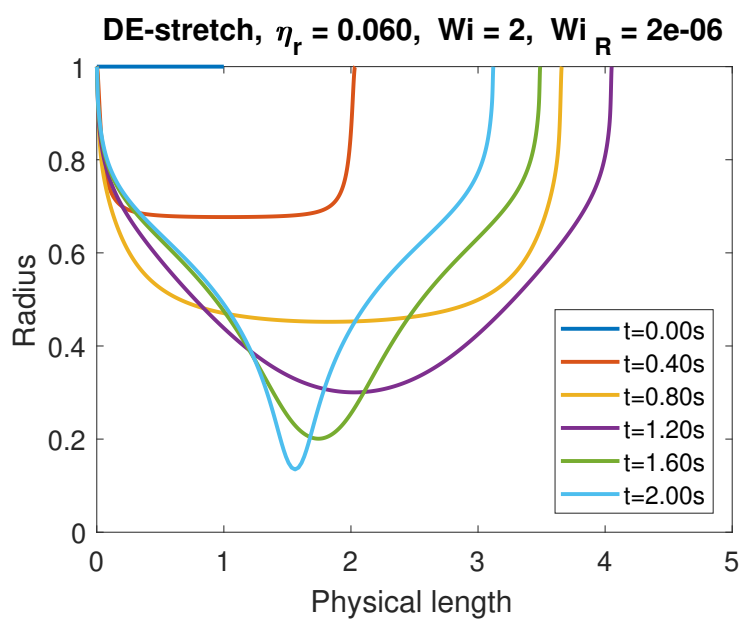

(b)

FIG. 7: Continuation of the slender filament simulation of controlled stretching of the single-mode DE-toy model in Fig. 6b. (a): True (top) and nominal (lower) Hencky strains as function of time. (b): Radial profiles for times 0.00, 0.40, 0.80, 1.20, 1.60, and 2.00, in units of $\tau_{d}$, which, for convenience, was set to $1 \mathrm{~s}$.

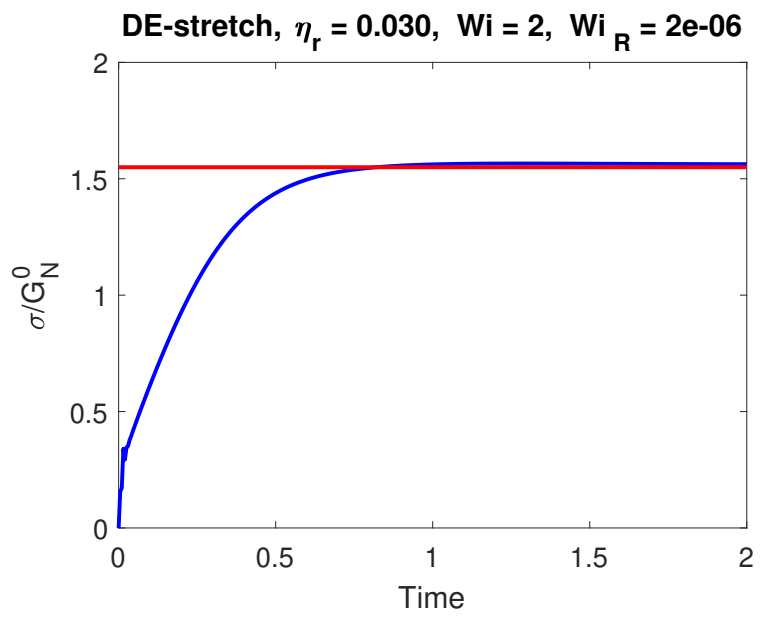

(a)

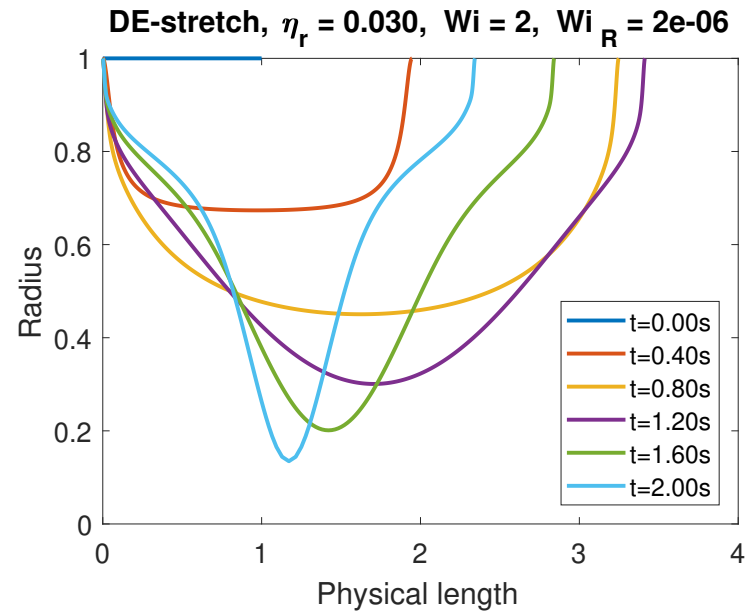

(b)

FIG. 8: Slender filament simulations of start-up of controlled stretching of DE-toy model at $\mathrm{Wi}_{R} \ll 1$ with continuous spectrum (Eqs. 8 to 11). (a): (blue curve) the extensional stress growth as function of time; (red line) the corresponding analytical steady stress. (b): Radial profiles for times $0.00,0.40,0.80,1.20,1.60$, and 2.00, in units of $\tau_{d}$, which, for convenience, was set to $1 \mathrm{~s}$. 
therefore, to ask if the non-stretching Doi-Edwards model (in the shape of the toy version) will perform in the same way. To investigate this, a simulation was performed with the DE-toy model at a vanishingly small stretch relaxation time.

Figure 6 illustrates the steady extensional stress of the single-mode (Eq. 7) DE-toy model as function of $\mathrm{Wi}=\dot{\epsilon} \tau_{d}$. Without solvent viscosity (full line) the stress saturates at about $\left(\sigma_{z z}-\sigma_{r r}\right) / G_{0} \approx 3.0$. The viscosity function is similar to that of the Rolie-Poly model in Fig. 4. Fig. $6 \mathrm{~b}$ shows the stress in units of $G_{0}$ as function of time in units of $\tau_{d}$. Also shown (in red) is the analytical steady stress. It appears that the steady stress is approached in less than one relaxation time. The corresponding true and nominal Hencky strains, as well as the radial profiles, are depicted in Fig. 7. Indeed the DE-toy model produces a behavior closely resembling that of the non-stretch Rolie-Poly model, possibly due to the stress saturation inherent in both models.

All constitutive equations considered by Hoyle and Fielding reduce in the linear viscoelastic limit to a single-mode Maxwell model augmented with a solvent viscosity. This leaves the question to what extent, for example, the elastic Considère criterion would be influenced by a broad linear viscoelastic spectrum. To answer this question, consider in Fig. 8 the results of a simulation in which the single-mode Maxwell model has been replaced by the continuous BSW spectrum. At the same time, the relative solvent viscosity has been reduced from $6 \%$ of the zero shear rate viscosity to $3 \%$. The resemblance of the radial profiles in Figs. $\mathbf{7 b}$ and $\mathbf{8} \mathbf{b}$ is remarkable. It appears that the effect of the BSW spectrum over the single-mode Maxwell spectrum is to effectively add a little extra relaxation that can be compensated by the addition of a small extra amount of solvent viscosity in the effective single-mode model.

\section{Relaxation of stress after start-up flow}

So far, it has been demonstrated that liquids with the property of stress saturation typically develop a localized neck when stretched at large rates. This has been illustrated both when the underlying constitutive equation is formulated in differential form and in integral form. The instability occurs both for a single Maxwell mode elasticity and with a continuum of relaxation times. In other words, the instability is a property of the non-linearity of the underlying constitutive equation more or less independent of the linear viscoelastic properties. Yet irrespective of the stress saturation, values of the steady viscosity can still be obtained, provided that the control scheme keeps the stretch rate in the mid-filament plane constant. On the other hand, simulations (not shown here) have demonstrated that no steady stress is obtained without a 
control scheme.

A final question relates to the action of molecular stretching as modeled by Eq. 15. This brings in the stretch Weissenberg number $\mathrm{Wi}_{R}=\dot{\epsilon} \tau_{s}$ in addition to $\mathrm{Wi}=\dot{\epsilon} \tau_{d}$. Several simulations have shown that molecular stretching, when it occurs, has a stabilizing effect on the filament. More specifically when Wi $<1$ no localized neck occurs because the elastic Considère mode is not excited. The regime $\mathrm{Wi}>1$ but $\mathrm{Wi}_{R}<1$ is the regime already explored in Figs. 5, 7 and 8. Finally, when $\mathrm{Wi}>1$ and $\mathrm{Wi}_{R}>1$ no localized necking occurs.

To illustrate the rather complex interaction between molecular orientation, stretching and relaxation, slender filament simulations have been performed, and the results are shown in Figs. 9 and 10. The material properties are inspired by (although not exactly equal to) measurements on narrow molar mass polystyrene ${ }^{37,42}$. The use of non-dimensional time is abandoned here. Thus $\dot{\epsilon}=0.03 \mathrm{~s}^{-1}$ (up to $100 \mathrm{~s}$ ) and time is in seconds. The time constants have been chosen to be $\tau=1000 \mathrm{~s}$ and (somewhat arbitrarily) $\tau_{s}=100 \mathrm{~s}$. The stress in Fig. 9 is in units of $G_{0}=250 \mathrm{kPa}$, while the radii in Fig. 10 are non-dimensionalized by the initial radius, which is used as the length scale. The simulations depict the start-up of constant stretch rate up to $100 \mathrm{~s}$ (i.e., $\epsilon=3$ ) and stress relaxation after that. ${ }^{42}$ The following features may be extracted:

- The molecular stretching has a strong stabilizing effect on the melt. In the absence of molecular stretching, as already demonstrated, it is not possible to follow the liquid filament beyond a Hencky strain of 2 (Figs. 5, 7 and 8). Here a nicely homogeneous filament is followed to $\epsilon=3$ as seen in Fig. 10a. Likewise, there is very little difference between the true and nominal Hencky strain up to 100 s, as seen in Fig. 9a.

- Secondly, it appears from Fig. 9b, that the steady stress is obtained after about $100 \mathrm{s.}$ This may be surprising in view of the (approximate) reptation time of $1000 \mathrm{~s}$, but it does, of course, correctly reflect the experimental observation ${ }^{37}$ that steady stress for polystyrene melts is achieved at around Hencky strains of $3-4$.

- Thirdly, the simulations reproduce the experimental observations ${ }^{42}$ that a considerable reverse motion of the plates is needed to keep the Hencky strain constant in the relaxation phase as seen in both Figs. 9a and 10a. The molecular mechanism for this phenomenon can be seen from Fig. 10b. The stabilization during the stretching phase is the result of a molecular stretch of about $\lambda=2.5$. But this stretching dies out after about $t=200 \mathrm{~s}$ (corresponding to about one stretch relaxation time after the termination of imposed 
stretching). The corresponding rapid decrease of stress is seen in Fig. 9 for time $t \in$ $(100 \mathrm{~s}, 200 \mathrm{~s})$. For $t>200 \mathrm{~s}$ the filament still has an uneven stress distribution, which would in the absence of the control scheme cause rapid failure of the filament ${ }^{43,44}$.

In the absence of control action, the filament will most likely fail under the development of localized necking. Such necking has been observed by Wang et al. ${ }^{43}$, simulated by Lyhne et $a l^{44}$, and extensively analyzed for a wide range of constitutive models by Hoyle and Fielding ${ }^{12}$.

The interest in performing stress relaxation experiments as simulated here is to study the molecular relaxation of highly stretched molecules in a quiescent liquid ${ }^{45-49}$. Such experiments can then be compared with corresponding coarse-grained Molecular Dynamics simulations that are now emerging ${ }^{50}$. The main conclusion here is that such experiments can indeed be performed, provided that an online control scheme is coupled to the rheometer.

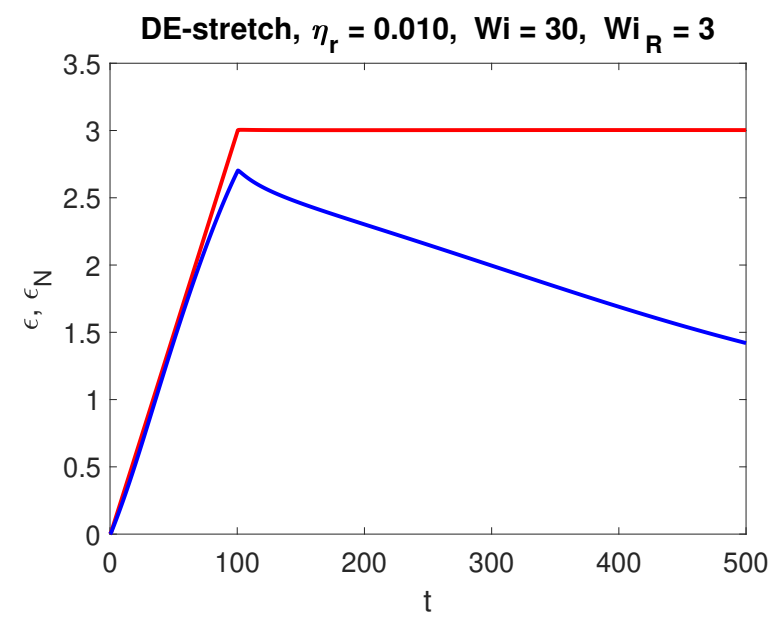

(a)

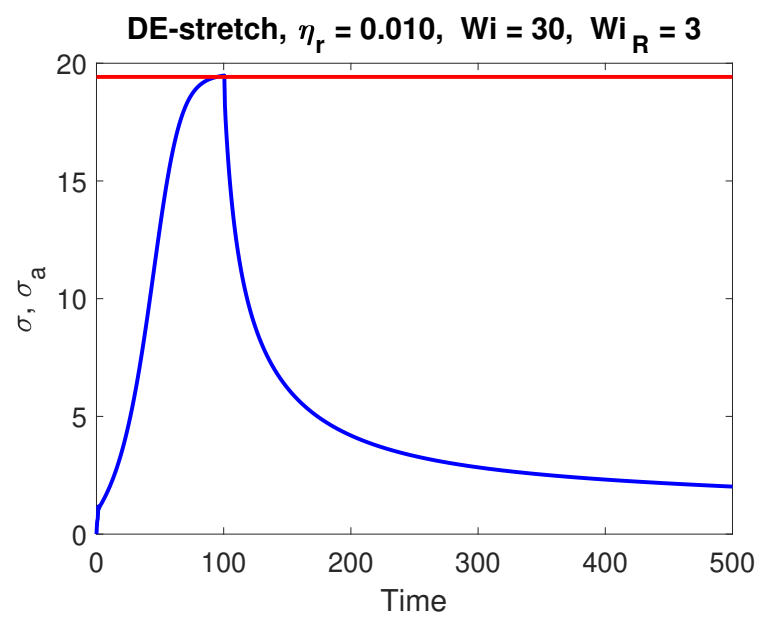

(b)

FIG. 9: Slender filament simulations of start-up followed by stress relaxation of single-mode DE-toy model. (a): True (top) and nominal (lower) Hencky strains as function of time. (b): Mid-filament stress (blue) and analytical expected steady stress (red). Time $t$ for both x-axes are in units of seconds.

\section{DISCUSSION AND CONCLUSIONS}

The simulations have shown that adjustment of the plate motion by online control is mandatory for accuracy in extensional rheometry by filament stretching. The only material for which control is not needed is the high Wi number stretching of an Oldroyd-B liquid. But that corresponds to the stretching of cross-linked natural rubber for which no viscosity can be expected. 


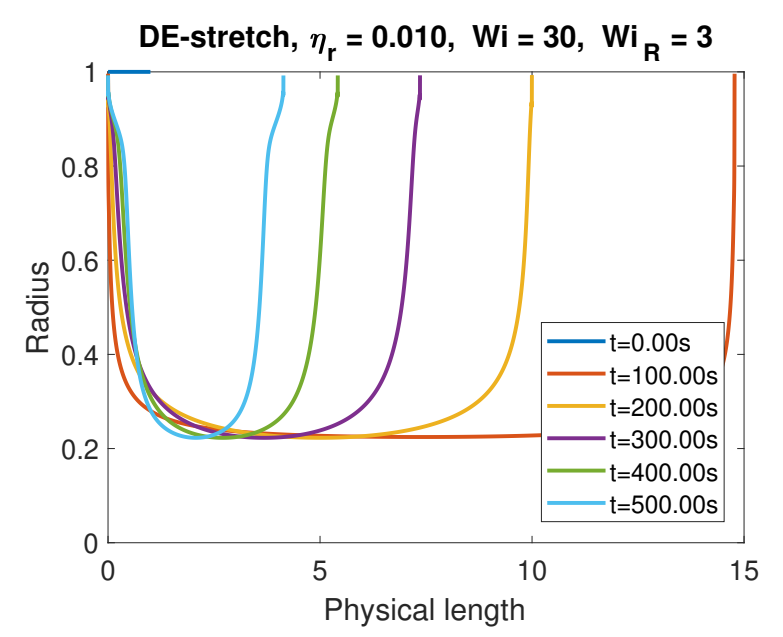

(a)

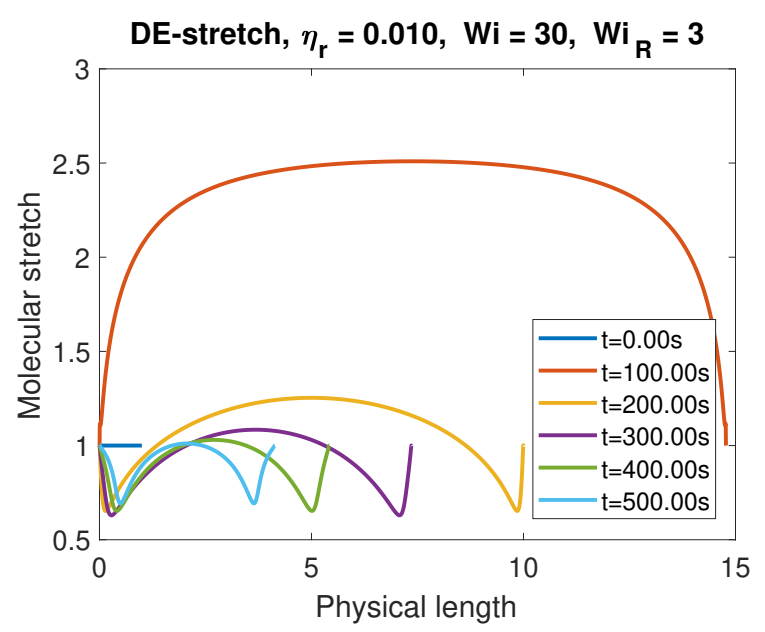

(b)

FIG. 10: Continuation of the slender filament simulation of controlled stretching of the single-mode DE-toy model in Fig. 9. (a): Radial profiles for time 1.0, 100.0, 200.0, 300.0, 400.0 and 500.0, in units of seconds. (b): Molecular stretch factor at each of the corresponding time point.

All other combinations of liquids and rates suffer from at least one of the Hoyle-Fielding instability modes. The stress-curvature mode appears to be quite mild, with possible stretching seemingly without a fluid mechanical termination. In practice, of course, the control loop will terminate, if not before then, when the filament diameter approaches the wavelength of the laser beam.

The dangerous mode is clearly the elastic Considère mode, which sets an upper limit on the Hencky strain achievable. For the stress saturating liquid models investigated here, this maximum is about two Hencky strain units. Fortunately, steady stress is achieved at about one Hencky strain unit, so it is possible to measure the steady viscosity even of such liquids.

The elastic Considère instability in linear polymers can be suppressed by stretching of the molecules from their equilibrium primitive path-length, i.e., at $\mathrm{Wi}_{R}>1$. This does leave a gap for the dangerous instability mode in the regime $\mathrm{Wi}_{R}<1<\mathrm{Wi}$ in which attempts to measure the stress-growth coefficient will terminate on the linear viscoelastic envelope in the absence of online control. Such a problem can be seen in earlier studies ${ }^{51}$. While molecular stretching prevents filament failure in the start-up of steady stretching, the molecular stretching appears to drive a dangerous instability in a subsequent relaxation part of an experiment. The simulation results presented here are in full agreement with the expected behavior from the analysis by Hoyle and Fielding ${ }^{12}$. Fortunately, adjustment of the plates enables reliable stress 
relaxation measurements to be obtained.

The present analysis (and the Hoyle-Fielding analysis) implicitly assumes that the deformation of the filament remains axis-symmetrical. However, it has been documented ${ }^{52,53}$ that complex liquids may undergo a rapid symmetry breaking fracture initiated from the filament edge. Also, in what amounts to controlled filament stretching measurements on wormlike micelles, Rothstein ${ }^{54}$ reported failure of the filaments before any significant necking has occurred at strains typically less than 3. While Rothstein did not have fast imaging, this too could well be such a fracture phenomenon. As a phenomenon distinct from necking, fracture is too fast and irreversible to be prevented by feedback control.

\section{Acknowledgements}

This work is to the memory of our professor R.B. Bird. We are very thankful for his influence directly on O.H. and thereby indirectly on Y.W. and Q.H.

\section{Data Availability Statement}

The data that supports the findings of this study are available within the article.

\section{Conflict of Interest}

The authors have no conflicts to disclose.

\section{APPENDIX A: EULERIAN IMPLEMENTATION}

The physical equations to be solved are the following ${ }^{10}$ :

$$
\begin{aligned}
\frac{\partial}{\partial t} A+V \frac{\partial}{\partial z} A & =-\frac{\partial V}{\partial z} A \\
\frac{\partial}{\partial z}\left(A \sigma_{E}\right) & =0 \\
\sigma_{E} & =G_{0} Z+3 \eta_{s} \frac{\partial V}{\partial z}
\end{aligned}
$$

Here, $A(z, t)$ and $V(z, t)$ are the physical area and velocity of the filament at position $z$ and time $t$. Moreover, $\eta_{s}$ is the Newtonian viscosity, and $G_{0}$ is the plateau modulus. $Z$ signifies the difference between the axial and radial components of the polymer conformation tensor. In the work of Hoyle and Fielding ${ }^{10}, Z$ is referred to as the viscoelastic conformation variable. It will be given by a viscoelastic constitutive equation. 
Returning to Eqs. A1, A2 and A3, we now apply change of variables ${ }^{10}$ as follows:

$$
\begin{aligned}
& u=z \exp \left(-\epsilon_{N}\right) \\
& v=V \exp \left(-\epsilon_{N}\right) \\
& a=A \exp \left(\epsilon_{N}\right)
\end{aligned}
$$

where the nominal Hencky strain, $\epsilon_{N}$ is given by Eq. 1. Introducing these transformations we get by the chain rule

$$
\begin{aligned}
& \frac{\partial}{\partial z}=\exp \left(-\epsilon_{N}\right) \frac{\partial}{\partial u} \\
& \frac{\partial}{\partial t}=-\dot{\epsilon}_{N} u \frac{\partial}{\partial u}+\frac{\partial}{\partial t}
\end{aligned}
$$

where $\dot{\epsilon}_{N}=d \epsilon_{N} / d t$ is the nominal Hencky strain rate. Consequently, the equations become

$$
\begin{aligned}
\frac{\partial a}{\partial t}+\left(v-\dot{\epsilon}_{N} u\right) \frac{\partial a}{\partial u} & =-\left(\frac{\partial v}{\partial u}-\dot{\epsilon}_{N}\right) a \\
\frac{\partial}{\partial u}\left(a \sigma_{E}\right) & =0 \\
\sigma_{E} & =G_{0} Z+\eta_{s} \frac{\partial v}{\partial u}
\end{aligned}
$$

Hoyle and Fielding ${ }^{10}$ formulated a generic model as follows:

$$
\frac{\partial Z}{\partial t}+\left(v-\dot{\epsilon}_{N} u\right) \frac{\partial Z}{\partial u}=\frac{\partial v}{\partial u} f(Z)-g(Z) / \tau
$$

Where for the toy Rolie-Poly model:

$$
\begin{aligned}
& f(Z)=2+2 Z-\beta Z^{2} \\
& g(Z)=Z
\end{aligned}
$$

To summarize, we solve for the following three functions:

$$
\begin{aligned}
& v=v(t, u) \\
& a=a(t, u) \\
& Z=Z(t, u)
\end{aligned}
$$

from the equations:

$$
\begin{aligned}
\frac{\partial a}{\partial t}+\left(v-\dot{\epsilon}_{N} u\right) \frac{\partial a}{\partial u} & =-\left(\frac{\partial v}{\partial u}-\dot{\epsilon}_{N}\right) a \\
\frac{\partial}{\partial u}\left[a\left(G_{0} Z+\eta_{s} \frac{\partial v}{\partial u}\right)\right] & =0 \\
\frac{\partial Z}{\partial t}+\left(v-\dot{\epsilon}_{N} u\right) \frac{\partial Z}{\partial u} & =\frac{\partial v}{\partial u} f(Z)-g(Z) / \tau
\end{aligned}
$$


With boundary conditions:

$$
\begin{aligned}
& v(t, 0)=0 \\
& v(t, 1)=\dot{\epsilon}_{N}
\end{aligned}
$$

and initial conditions on $a$ and $Z$ in the form of a possible pre-stretch on $a$ and equilibrium on $Z$ :

$$
\begin{aligned}
& a(0, u)=a_{0}[1-\alpha u(1-u)] \\
& Z(0, u)=0
\end{aligned}
$$

where $a_{0}$ is a constant and $\alpha$ is a pre-stretch factor. The no-slip conditions at the plates are not specified directly but approximated by the method of Stokes et al. ${ }^{55}$.

\section{APPENDIX B: LAGRANGIAN IMPLEMENTATION}

The basic variables in the Lagrangian specification are time and material coordinates. Time is discretized into $n t$ equally spaced values. The filament is given by the initial coordinates of $n z$ equally spaced particles along the filament. The total transmitted force (the "engineering stress") in the filament is unknown. A simulation then consists of following these particles and the force as a function of time. In addition, a molecular stretch $\lambda$ may be defined in each of the $n z-1$ segments between material particles. For an illustration of the principle, it suffices to summarize the variables and equations in the absence of stretch variables. We have: Time:

$$
t(j)=j d t, j=1,2, \cdots n t
$$

Material coordinates:

$$
z(i, j), i=1,2, \cdots n z
$$

Boundary conditions

$$
\begin{gathered}
z(1, j)=0, j=1,2 \cdots n t \\
z(n z, j)=f(t(j)), j=1,2 \cdots n t
\end{gathered}
$$

where, for example, $f(\ldots)=\exp \left(\dot{\epsilon}_{0} t\right)$ or any other described function. The force (per area) transmitted in the filament, $F / A_{0}$, is an unknown, and therefore, at any one time $(j)$, we have $n z+1$ unknowns, let them be $X$, where

$$
\begin{aligned}
X(i) & =z(i), i=1,2, \cdots n z \\
X(n z+1) & =F / A_{0}
\end{aligned}
$$


Hence we need $n z+1$ equations in the form $F(i)=0, i=1,2, \cdots, n z+1$. These can be symbolically written in the form of

$$
\begin{aligned}
F(1) & =X(1) \\
F(i+1) & =\text { force in element } \mathrm{i}-X(n z+1), i=1,2, \cdots, n z-1 \\
F(n z+1) & =X(n z)-f(t(j))
\end{aligned}
$$

The no-slip conditions at the plates are not specified directly but approximated by the method of Stokes et al. ${ }^{55}$. The equations $F(i)=0$ are solved by Newton's iteration method at each time step. Quadratic convergence is observed to machine accuracy.

\section{REFERENCES}

${ }^{1}$ R. B. Bird, R. C. Armstrong, and O. Hassager, Dynamics of Polymeric Liquids. Vol. I: Fluid Mechanics, 2nd ed. (John Wiley \& Sons, New York, 1987).

${ }^{2}$ R. B. Bird, C. F. Curtiss, R. C. Armstrong, and O. Hassager, Dynamics of Polymeric Liquids. Vol. II: Kinetic Theory, 2nd ed. (John Wiley \& Sons, New York, 1987).

${ }^{3}$ G. Ianniruberto, G. Marrucci, and Y. Masubuchi, "Melts of linear polymers in fast flows," Macromolecules 53, 5023-5033 (2020).

${ }^{4}$ M. Zatloukal and J. Drabek, "Reduction of monomeric friction coefficient for linear isotactic polypropylene melts in very fast uniaxial extensional flow," Phys. Fluids 33, 051703 (2021) .

${ }^{5}$ J. Eggers and E. Villermaux, "Physics of liquid jets," Rep. Prog. Phys. 71, 036601 (2008).

${ }^{6}$ O. Hassager, M. I. Kolte, and M. Renardy, "Failure and nonfailure of fluid filaments in extension," J. Non-Newton. Fluid Mech. 76, 137-151 (1998).

${ }^{7}$ A. Ya. Malkin and C. J. S. Petrie, "Some conditions for rupture of polymer liquids in extension," J. Rheol. 41, 1-25 (1997).

${ }^{8}$ X. Zhu and S.-Q. Wang, "Mechanisms for different failure modes in startup uniaxial extension: Tensile (rupture-like) failure and necking," J. Rheol. 57, 223-248 (2013).

${ }^{9}$ A. C. Papanastasiou, L. E. Scriven, and C. W. Macosko, "An integral constitutive equation for mixed flows: viscoelastic characterization," J. Rheol. 27, 387-410 (1983).

${ }^{10}$ D. M. Hoyle and S. M. Fielding, "Criteria for extensional necking instability in complex fluids and soft solids. Part I: Imposed hencky strain rate protocol," J. Rheol. 60, 1347-1375 (2016).

${ }^{11}$ D. M. Hoyle and S. M. Fielding, "Criteria for extensional necking instability in complex fluids 
and soft solids. Part II: Imposed tensile stress and force protocols," J. Rheol. 60, 1377-1397 (2016).

${ }^{12}$ D. M. Hoyle and S. M. Fielding, "Necking after extensional filament stretching of complex fluids and soft solids," J. Non-Newton. Fluid Mech. 247, 132-145 (2017).

${ }^{13}$ T. Sridhar, V. Tirtaatmadja, D. A. Nguyen, and R. K. Gupta, "Measurement of extensional viscosity of polymer solutions," J. Non-Newton. Fluid Mech. 40, 271-280 (1991).

${ }^{14} \mathrm{~V}$. Tirtaatmadja and T. Sridhar, "Comparison of constitutive equations for polymer solutions in uniaxial extension," J. Rheol. 39, 1133-1160 (1995) .

${ }^{15}$ G. H. McKinley and T. Sridhar, "Filament-stretching rheometry of complex fluids," Annu. Rev. Fluid Mech. 34, 375-415 (2002).

${ }^{16}$ A. Bach, H. K. Rasmussen, and O. Hassager, "Extensional viscosity for polymer melts measured in the filament stretching rheometer," J. Rheol. 47, 429-441 (2003).

${ }^{17}$ T. Sridhar, M. Acharya, D. A. Nguyen, and P. K. Bhattacharjee, "On the extensional rheology of polymer melts and concentrated solutions," Macromolecules 47, 379-386 (2014).

${ }^{18}$ P. K. Bhattacharjee, D. A. Nguyen, Y. Masubuchi, and T. Sridhar, "Extensional step strain rate experiments on an entangled polymer solution," Macromolecules 50, 386-395 (2017).

${ }^{19}$ N. V. Orr and T. Sridhar, "Probing the dynamics of polymer solutions in extensional flow using step strain rate experiments," J. Non-Newton. Fluid Mech. 82, 203-232 (1999).

${ }^{20}$ S. L. Anna, C. Rogers, and G. H. McKinley, "On controlling the kinematics of a filament stretching rheometer using a real-time active control mechanism," J. Non-Newton. Fluid Mech. 87, 307-335 (1999).

${ }^{21}$ J. M. R. Marín, J. K. Huusom, N. J. Alvarez, Q. Huang, H. K. Rasmussen, A. Bach, A. L. Skov, and O. Hassager, "A control scheme for filament stretching rheometers with application to polymer melts," J. Non-Newton. Fluid Mech. 194, 14-22 (2013).

${ }^{22}$ S. L. Anna and G. H. McKinley, "Elasto-capillary thinning and breakup of model elastic liquids," J. Rheol. 45, 115-138 (2001).

${ }^{23}$ A. Mohammad Karim, "Experimental dynamics of newtonian and non-newtonian droplets impacting liquid surface with different rheology," Phys. Fluids 32, 043102 (2020).

${ }^{24}$ M. S. N. Oliveira, R. Yeh, and G. H. McKinley, "Iterated stretching, extensional rheology and formation of beads-on-a-string structures in polymer solutions," J. Non-Newton. Fluid Mech. 137, 137-148 (2006).

${ }^{25}$ A. Mohammad Karim, W. J. Suszynski, W. B. Griffith, S. Pujari, L. F. Francis, and M. S. Carvalho, "Effect of viscoelasticity on stability of liquid curtain," J. Non-Newton. Fluid Mech. 
257, 83-94 (2018) .

${ }^{26}$ This definition implicitly assumes incompressibility which is a good assumption for most soft materials.

${ }^{27}$ Q. Huang, J. Ahn, D. Parisi, T. Chang, O. Hassager, S. Panyukov, M. Rubinstein, and D. Vlassopoulos, "Unexpected stretching of entangled ring macromolecules," Phys. Rev. Lett. 122, 208001 (2019).

${ }^{28}$ M. Yao, S. H. Spiegelberg, and G. H. McKinley, "Dynamics of weakly strain-hardening fluids in filament stretching devices," J. Non-Newton. Fluid Mech. 89, 1-43 (2000).

${ }^{29}$ R. S. Graham, A. E. Likhtman, T. C. B. McLeish, and S. T. Milner, "Microscopic theory of linear, entangled polymer chains under rapid deformation including chain stretch and convective constraint release," J. Rheol. 47, 1171-1200 (2003).

${ }^{30}$ A. E. Likhtman and R. S. Graham, "Simple constitutive equation for linear polymer melts derived from molecular theory: Rolie-poly equation," J. Non-Newton. Fluid Mech. 114, 1-12 (2003).

${ }^{31}$ There is an extra linear term in the non-stretchable model that is not included here. We include the quadratic term, which is the main point.

${ }^{32}$ M. Doi and S. F. Edwards, The Theory of Polymer Dynamics (Oxford University Press, New York, 1986).

${ }^{33}$ Numerical results for the linearized necking dynamics of the non-stretch Rolie-Poly model are shown in Fig. 3 (d) of Ref. ${ }^{10}$ by Hoyle and Fielding. .

${ }^{34}$ M. S. Bazzi and M. S. Carvalho, "Effect of viscoelasticity on liquid sheet rupture," J. NonNewton. Fluid Mech. 264, 107-116 (2019).

${ }^{35} \mathrm{M}$. Baumgaertel, A. Schausberger, and H. H. Winter, "The relaxation of polymers with linear flexible chains of uniform length," Rheol. Acta 29, 400-408 (1990).

${ }^{36} \mathrm{~J}$. K. Jackson and H. H, Winter, "Entanglement and flow behavior of bidisperse blends of polystyrene and polybutadiene," Macromolecules 28, 3146-3155 (1995).

${ }^{37}$ Q. Huang, O. Mednova, H. K. Rasmussen, N. J. Alvarez, A. L. Skov, K. Almdal, and O. Hassager, "Concentrated polymer solutions are different from melts: Role of entanglement molecular weight," Macromolecules 46, 5026-5035 (2013).

${ }^{38}$ Ad Hoc Committee on Official Nomenclature and Symbols, "Official symbols and nomenclature of the society of rheology," J. Rheol. 57, 1047-1055 (2013).

${ }^{39}$ D. S. Pearson, A. D. Kiss, L. J. Fetters, and M. Doi, "Flow-induced birefringence of concentrated polyisoprene solutions," J. Rheol. 33, 517-535 (1989). 
${ }^{40}$ E. Narimissa and M. H. Wagner, "Review on tube model based constitutive equations for polydisperse linear and long-chain branched polymer melts," J. Rheol. 63, 361-375 (2019).

${ }^{41}$ R. J. Blackwell, T. C. B. McLeish, and O. G. Harlen, "Molecular drag-strain coupling in branched polymer melts," J. Rheol. 44, 121-136 (2000).

${ }^{42}$ J. K. Nielsen, H. K. Rasmussen, and O. Hassager, "Stress relaxation of narrow molar mass distribution polystyrene following uniaxial extension," J. Rheol. 52, 885-899 (2008).

${ }^{43}$ Y. Wang, P. Boukany, S.-Q. Wang, and X. Wang, "Elastic breakup in uniaxial extension of entangled polymer melts," Phys. Rev. Lett. 99, 237801 (2007).

${ }^{44}$ A. Lyhne, H. K. Rasmussen, and O. Hassager, "Simulation of elastic rupture in extension of entangled monodisperse polymer melts," Phys. Rev. Lett. 102, 138301 (2009).

${ }^{45}$ Q. Huang, H. K. Rasmussen, A. L. Skov, and O. Hassager, "Stress relaxation and reversed flow of low-density polyethylene melts following uniaxial extension," J. Rheol. 56, 1535-1554 (2012).

${ }^{46}$ L. Hengeller, Q. Huang, A. Dorokhin, N. J. Alvarez, K. Almdal, and O. Hassager, "Stress relaxation of bi-disperse polystyrene melts," Rheol. Acta 55, 303-314 (2016).

${ }^{47}$ Q. Huang, S. Agostini, L. Hengeller, M. Shivokhin, N. J. Alvarez, L. R. Hutchings, and O. Hassager, "Dynamics of star polymers in fast extensional flow and stress relaxation," Macromolecules 49, 6694-6699 (2016).

${ }^{48}$ Q. Huang and H. K. Rasmussen, "Stress relaxation following uniaxial extension of polystyrene melt and oligomer dilutions," J. Rheol. 60, 465-471 (2016).

${ }^{49}$ Q. Huang, S. Costanzo, C. Das, and D. Vlassopoulos, "Stress growth and relaxation of dendritically branched macromolecules in shear and uniaxial extension," J. Rheol. 61, 35-47 (2017).

${ }^{50}$ T. C. O'Connor, A. Hopkins, and M. O. Robbins, "Stress relaxation in highly oriented melts of entangled polymers," Macromolecules 52, 8540-8550 (2019).

${ }^{51}$ D. Auhl, P. Chambon, T. C. B. McLeish, and D. J. Read, "Elongational flow of blends of long and short polymers: Effective stretch relaxation time," Phys. Rev. Lett. 103, 136001 (2009).

${ }^{52}$ H. Tabuteau, S. Mora, G. Porte, M. Abkarian, and C. Ligoure, "Microscopic mechanisms of the brittleness of viscoelastic fluids," Phys. Rev. Lett. 102, 155501 (2009).

${ }^{53}$ Q. Huang, N. J. Alvarez, A. Shabbir, and O. Hassager, "Multiple cracks propagate simultaneously in polymer liquids in tension," Phys. Rev. Lett. 117, 087801 (2016).

${ }^{54}$ J. P. Rothstein, "Transient extensional rheology of wormlike micelle solutions," J. Rheol. 47, 
$1227-1247$ (2003).

${ }^{55}$ Y. M. Stokes, E. O. Tuck, and L. W. Schwartz, "Extensional fall of a very viscous fluid drop," Q. J. Mech. Appl. Math. 53, 565-582 (2000).

${ }^{56}$ Q. Huang and H. K. Rasmussen, "Extensional flow dynamics of polystyrene melt," J. Rheol. 63, 829-835 (2019). 